The Astrophysical Journal Supplement Series, 90:827-831, 1994 February

(C) 1994. The American Astronomical Society. All rights reserved. Printed in U.S.A.

\title{
UNPULSED HIGH-ENERGY RADIATION FROM THE CRAB PULSAR AND NEBULA
}

\author{
W. M. Cheung AND K. S. Cheng \\ Department of Physics, The University of Hong Kong, Pokfulam Road, Hong Kong \\ Received 1993 February 11; accepted 1993 May 25
}

\begin{abstract}
Generally, people believe that the unpulsed high-energy gamma rays from the direction of Crab Nebula and pulsar comes from the nebula. But it is entirely possible that the unpulsed high-energy gamma rays from the direction of the Crab Nebula and pulsar are actually emitted from a region extending to a couple of light cylinder radii from the pulsar instead of from the extensive nebula. In this conference paper, we study the possibility that the unpulsed high-energy gamma rays from $100 \mathrm{MeV}$ to $10 \mathrm{GeV}$ are emitted from the extensive nebula. In our model, two pulsed photon beams from two different outer gaps cross each other beyond the light cylinder and result in pair production. Since the pitch angles of these pairs do not correlate with the local magnetic field, and the typical mean free path for pair production is comparable to the local radius of curvature, the subsequent synchrotron radiation and inverse-Compton scattering produce unpulsed X-rays and gamma-rays respectively.
\end{abstract}

Subject headings: acceleration of particles — gamma rays: theory — ISM: individual (Crab Nebula) pulsars: individual (Crab) - radiation mechanisms: nonthermal

\section{INTRODUCTION}

The angular resolution of telescopes and detectors in radio, optical, and X-rays is good enough to separate the radiation of the Crab Nebula from that of the pulsar. However, the angular resolution of gamma-ray detectors does not allow one to separate the nebula from the pulsar in the Crab. It is generally assumed that the steady emission comes from the nebula and the pulsed radiation from the pulsar. As early as 1965 , a canonical model (Compton-synchrotron model) was proposed by Gould which accounts for the steady emission of the nebula. It suggested that the existence of relativistic electrons in the nebula will emit photons (IR-X-rays) through synchrotron radiation, and that these same photons are, in turn, boosted to highenergy photons $(\mathrm{TeV})$ by inverse-Compton scattering (ICS) with the relativistic electrons. The model was further refined by Rieke \& Weekes (1969) and by Grindlay \& Hoffmann (1971). This model successfully explains the observed spectrum from IR to $\mathrm{X}$-rays and the $\mathrm{TeV}$ region but not the energy range of $\operatorname{COS} B(50 \mathrm{MeV}-3 \mathrm{GeV})$. It is because, in the synchrotron-Compton model, the energy ranges of the synchrotron spectrum and the inverse-Compton spectrum depend on the maximum energy of the relativistic electrons which is limited either by the energy loss or diffusion loss. Kennel \& Coroniti (1984), by applying MHD flow models of the nebula, showed that because of the synchrotron radiation loss the maximum electron energy is about $10^{15} \mathrm{eV}$ which implies a synchrotron upper cutoff around a few $\mathrm{MeV}$ and results in an ICS spectrum which is too flat in this energy range.

More recently, De Jager \& Harding ( 1992) have reexamined the inverse-Compton scattering model of the Crab Nebula, using the magnetic field distribution derived from MHD flow models of the nebula (Coroniti 1990). They propose that the maximum electron energies could be up to $10^{16} \mathrm{eV}$, possibly accelerated at the shock in the pulsar wind. They argue that the synchrotron energy loss of the electrons is much weaker than the earlier calculation because the magnetic field in the shock acceleration region due to the partial annihilation of unequally striped field lines (Coroniti 1990) is much weaker than the average strength of the magnetic field in the nebula. They predict that the synchrotron spectrum will range from IR up to a few $\mathrm{GeV}$ and that the ICS spectrum takes over from a few $\mathrm{GeV}$ to $10^{16} \mathrm{eV}$. Their model spectrum agrees with the observed data nicely. However, there is still one unsatisfactory point about this model, namely, how could electrons be accelerated up to $10^{16} \mathrm{eV}$ ? De Jager \& Harding have addressed this question and have suggested that the acceleration mechanisms are either diffusive shock acceleration, simple electrostatic acceleration by drifting through a potential drop along the shock or turbulence acceleration (Arons et al. 1991). Nevertheless, all these mechanisms contain uncertainties. For the diffusive shock, the diffusion coefficient $D$, which plays an essential role in determining the maximum electron energy, must be solved for self-consistently. For the electrostatic acceleration, it is not clear why the numerous $e^{ \pm}$pairs cannot screen out the static electric field. Finally, the turbulence acceleration so far has been done in only one-dimensional particle simulations; it is not clear how it works in more realistic two-dimensional or three-dimensional cases. Therefore an alternative model may be necessary.

On the other hand, by using the outer magnetospheric gap model (Cheng, Ho, \& Ruderman 1986a, b; and Ho 1989, hereafter outer gap model), which was proposed to explain the pulsed radiation from the Crab pulsar, a model of unpulsed very high energy gamma rays from the Crab Nebula and pulsar can be constructed (Kwok, Cheng, \& Lau 1991, hereafter Paper I). It is an alternative mechanism in which the steady emission of very high energy gamma rays could come from a compact region, a couple of light cylinder radii beyond the pulsar. Similarly, the steady emission of low-energy (MeV-20 $\mathrm{GeV}$ ) gamma rays can be explained by using the same model 
(Cheung \& Cheng 1993, hereafter Paper II). In this conference paper, we will review these alternative unpulsed models.

This paper is organized as follows. In $\S 2$, we review the relavent material of the outer gap model and the model of unpulsed VHE gamma rays from the Crab pulsar. In $\S 3$, the production mechanisms and processes of the unpulsed gamma rays are presented. In $\S 4$, a detailed calculation of the unpulsed gamma rays is given. Finally, a comparison of our model results, the observed data, and the results of the synchrotronCompton model are given in $\S 5$.

\section{OUTER GAP MODEL AND UNPULSED VHE GAMMA-RAYS MODEL}

In outer gap models, it is assumed that global current flow in the outer magnetosphere of a spinning magnetized neutron star results in a specially located outer magnetosphere gap with large charge depletion (cf. Fig. 1). Within the gap, $\boldsymbol{E} \cdot \boldsymbol{B} \neq 0$. A plasma of $e^{ \pm}$will be created whenever the potential drop of the outer gap is sufficiently large to support the production of $\gamma$ rays of high enough energy to materialize as pairs, and thus limit the growth of the gap. The gap results in a slablike vol- ume. This slablike gap can act as an ultrarelativistic particle accelerator. The mechanisms of the interaction between photons, electrons, or positrons in the magnetosphere of the Crab Nebula are shown below.

Inside the outer gap, "gap" primary electrons and positrons are oppositely accelerated to extreme relativistic energies, which are limited at $20 \mathrm{TeV}$ by radiation reaction (N.B.: we use "gap" to differentiate $e^{ \pm}$pairs and photons produced inside the light cylinder from those created outside it). They lose most of their energies $\left(\sim 10^{15} \mathrm{eV}\right)$ via curvature radiation to photons with typical energies $\sim 20 \mathrm{GeV}$. These curvature $\gamma$ rays (gap primary photons) convert to $e^{ \pm}$pairs in collisions with $\mathrm{X}$-rays. These $\mathrm{X}$-rays come from the synchrotron emission from such (gap) secondary pairs created beyond the outer gap but within the light cylinder. Inverse-Compton scattering of the gap secondary pairs on the gap secondary X-rays boosts a fraction to $\gamma$-rays. This combination of the synchrotron spectrum and inverse-Compton scattering spectrum is proposed to account for the observed pulsed emission of the Crab pulsar from IR-optical to $10 \mathrm{GeV}$. The main reasons the gap secondary photons remain pulsed are $(a)$ the magnetic field inside the light cylinder can be well approximated by a dipole form and is also sufficiently strong to restrict the motion of the gap elec-

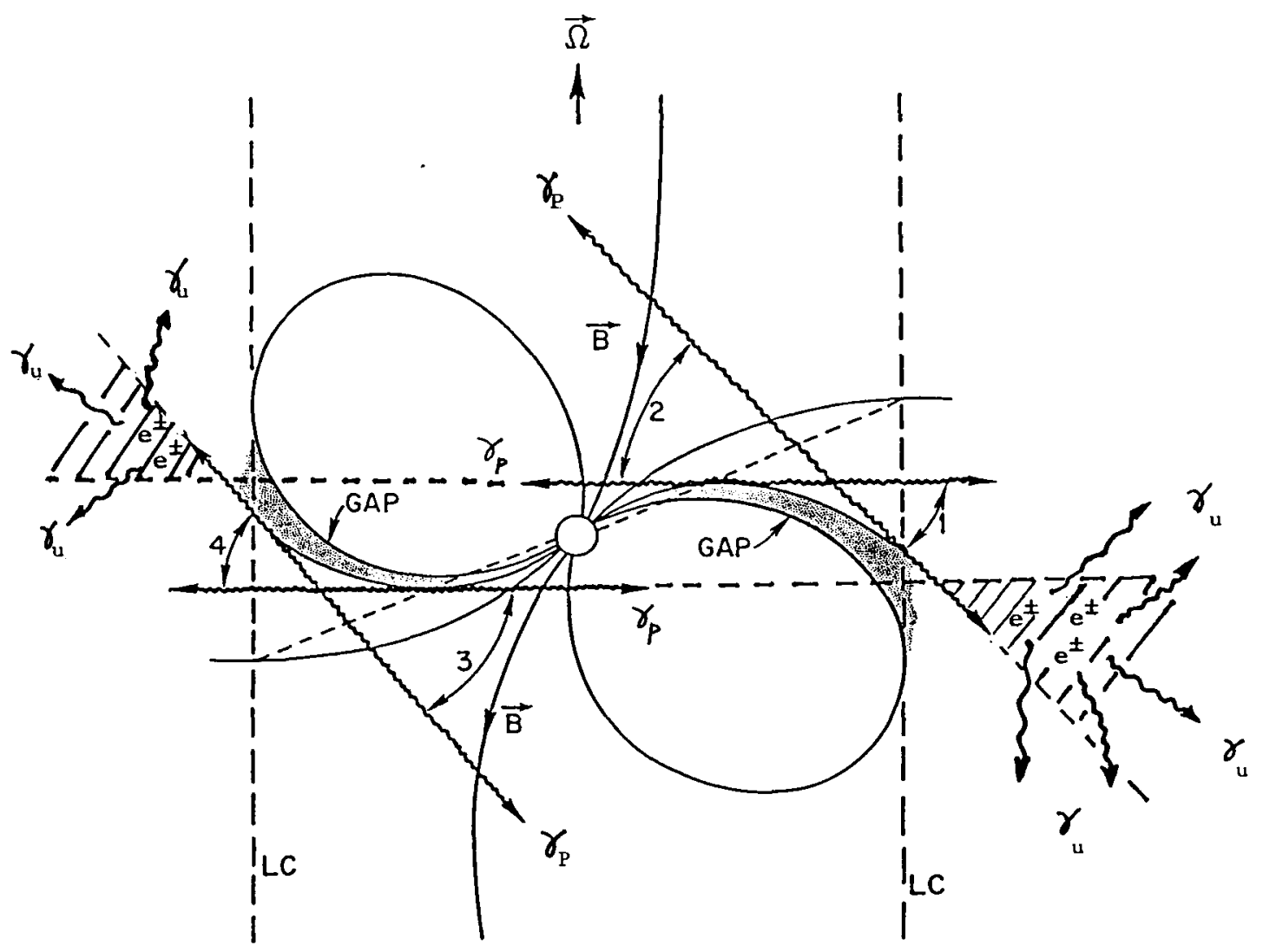

FIG. 1.-Schematic representation of the pair production and radiation processes beyond the light cylinder (LC) where the corotation speed equals the velocity of light. The shaded regions are the locations of outer gap where gap primary electrons and positrons are accelerated and lose their energies to high-energy curvature photons. These curvature $\gamma$-rays (gap primary photons) convert to (gap) secondary $e^{ \pm}$pairs in collisions with X-rays which come from the synchrotron emission from the same (gap) secondary pairs created beyond the outer gap. Four pulsed $\gamma$-ray beams $\left(\gamma_{p}\right)$ are emitted by these gap secondary pairs via synchrotron radiation and inverse-Compton scattering processes. Beyond the light cylinder, beam $1(2)$ and beam $3(4)$ will cross over each other ( hatched regions) and result in secondary $e^{ \pm}$pairs with large pitch angles. The subsequent radiation of these secondary pairs results in unpulsed $\gamma$-rays $\left(\gamma_{u}\right)$ in the energy ranges of X-ray and medium-energy $\gamma$-rays. 
trons/positrons to move roughly along the field lines and $(b)$ the pitch angles of the pairs $\left(\theta \leq 10^{-1}\right)$ are shown to be small (Cheng, Ho, \& Ruderman 1986a, b; Ho 1989).

Beyond the light cylinder, where the radius of curvature is much larger than $10^{8} \mathrm{~cm}$, the curvature radiation becomes unimportant and the gap primary positrons (electrons), if $\boldsymbol{\Omega} \cdot \mathbf{B}>0(\boldsymbol{\Omega} \cdot \mathbf{B}<0)$, can maintain their energies. Eventually, the gap primary positrons (electrons) from the first outer gap will inevitably collide with the gap IR photons from the second outer gap and produce $10 \mathrm{TeV}$ primary VHE $\gamma$-rays and vice versa (cf. Fig. 1). These primary VHE $\gamma$-rays are sufficiently energetic to produce secondary $e^{ \pm}$pairs by collision with the same pulsed IR photons. Since the mean free path of these conversion processes is comparable to the local radius of curvature, the pitch angles of the secondary pairs are large enough to result in isotropic secondary VHE $\gamma$-rays with typical energies $\sim \mathrm{TeV}$. The model results and the observed data have been compared in Paper $\mathbf{I}$.

\section{THE MODEL OF UNPULSED GAMMA-RAY EMISSION}

In the previous section, we noted that unpulsed VHE $\gamma$-rays from the direction of the Crab Nebula and pulsar may come from a compact region, in fact, just a couple of light cylinder radii away from the pulsar. We believe that the unpulsed $\gamma$ rays in the energy range of $50 \mathrm{MeV}-10 \mathrm{GeV}$ could also be produced in a similar crossover region. However, it is not the extreme relativistic gap primary electrons/positrons that play the roles at this time. Instead, when those part of the gap secondary $\gamma$-rays produced near the light cylinder propagate beyond the light cylinder, they will collide with the gap secondary photons emitted from another outer gap (cf. Fig. 1) and vice versa. The resulting secondary $e^{ \pm}$pairs can lose their energies via synchrotron radiation, and inverse-Compton scattering with the same gap secondary photons. These pair production and radiation mechanisms will continue until the optical depth becomes much less than unity.

Qualitatively, we could argue that these $\gamma$-rays produced beyond the light cylinder should be emitted isotropically. There are two main reasons. First, the magnetic field inside the light cylinder has a dipole form but it will gradually transform to a swirling magnetic field beyond the light cylinder. The gap secondary photons are emitted tangentially to the local magnetic field lines inside the light cylinder but the secondary pairs created by collisions with the gap $\mathrm{X}$-rays can make arbitrary pitch angles with the local magnetic field lines, because the locations of where photons are emitted and where they materialize to pairs are not necessarily correlated. Second, the mean free path of pair production, estimated to be $\sim 2 \times 10^{8} \mathrm{~cm}$, is not small compared with $\langle r\rangle \sim 3 r_{\mathrm{Ic}}$ which is the mean distance to the crossover region (Paper I).

\section{CALCULATION OF THE UNPULSED GAMMA-RAY SPECTRUM}

Inside the crossover region, $e^{ \pm}$pairs are produced when the gap $\gamma$-rays coming from one outer gap collide with the gap $X$-rays coming from the other outer gap. Before calculating the $e^{ \pm}$spectrum, the amount of pulsed gamma rays absorbed and surviving, denoted by $F_{p}^{\text {abs }}$ and $F_{p}^{\text {sur }}$, respectively, in this process should be calculated first (Blumenthal \& Gould 1970).
After the collision of these pulsed photon beams, which come from different regions, an $e^{ \pm}$pair will be produced. In turn, these $e^{ \pm}$pairs will produce the primary isotropic X-rays through synchrotron radiation, or gamma rays through inverse-Compton scattering with infrared-optical photons. It is worthwhile to calculate the energy distribution $d N\left(E_{e}\right) / d E_{e}$ of the $e^{ \pm}$pairs with energy $E_{e}$ (Blumenthal \& Gould 1970).

Once the spectrum of the secondary pairs is found, the secondary gamma-ray spectrum of synchrotron radiation and inverse-Compton scattering can readily be calculated (Blumenthal \& Gould 1970).

It should be noted that the unpulsed gamma rays produced at a distance of $\langle r\rangle=3 r_{\mathrm{Ic}}$ (hereafter $\left.\langle r\rangle_{1}\right)$, where $r_{\mathrm{Ic}}(=1.5 \times$ $10^{8} \mathrm{~cm}$ ) is the light cylinder radius, from the pulsar are the first generation of unpulsed gamma rays. However, further absorption of these photons at a distance of $\langle r\rangle_{i}$ from the pulsar produces the $i$ th generation of the unpulsed gamma rays. Further pair production processes are essentially the same as those of the first generation but with differences in the following input parameters:

$$
\begin{array}{r}
\text { (1) }\langle r\rangle_{i}=\langle r\rangle_{i-1}+\lambda_{i-1} \text { for } i>1 \\
\text { (2) } Q_{i}\left(E^{\prime}\right)=2\left(1-e^{-\tau_{i}\left(E_{\gamma}\right)}\right) F_{u_{i-1}}\left(E_{e}^{\prime}=E_{\gamma} / 2\right) \\
\text { for } i \geq 1
\end{array}
$$

where the subscript $i$ denotes the $i$ th generation of unpulsed gamma rays, and $\lambda_{i-1}$ is the mean free path of the $(i-1)$ th generation of $e^{ \pm}$pairs; and $F_{u_{i}}\left(E_{e}^{\prime}\right)$ is the $i$ th generation of the unpulsed gamma-ray spectrum for $i \geq 1$ with $F_{u_{0}}\left(E_{e}^{\prime}\right)=F_{p}$.

The observed unpulsed gamma-ray spectrum $F_{\text {tot }}$ is the sum of all the generations of the survived unpulsed low-energy gamma-ray spectra produced by synchrotron radiation and inverse-Compton scattering. That is,

$$
F_{\mathrm{tot}}\left(E_{\gamma}\right)=\sum_{i=1}^{\infty} F_{\mathrm{tot}_{i}}\left(E_{\gamma}\right) e^{-\tau_{i}\left(E_{\gamma}\right)} .
$$

Finally, the photon flux observed on Earth is $F_{\text {tot }}\left(E_{\gamma}\right) / 4 \pi D^{2}$, where $D$ is the distance of the Crab from Earth.

\section{DISCUSSION}

In calculating the unpulsed low-energy gamma-ray spectrum, we need to specify the two parameters $\Delta \Omega$, which are the solid angle of the pulsed radiation, and $\langle r\rangle_{1}$. However, as the geometry and the production mechanisms used in this paper are similar to those of Paper I, the values of the above two parameters are chosen the same as in Paper I. We start the calculation from the theoretical spectrum of the pulsed electromagnetic emission from the Crab pulsar (Cheng, Ho, \& Ruderman 1986a, b). Besides calculating the unpulsed gammaray spectrum, the theoretical spectrum of the pulsed electromagnetic emission is also modified (cf. Fig. 2). The modification is due to the redistribution of the pulsed gamma rays, mainly those with energy greater than $500 \mathrm{MeV}$, into unpulsed gamma rays.

Figure 3 shows the calculated unpulsed low-energy gammaray spectrum. Although both synchrotron radiation and inverse-Compton scattering contribute on it, they dominate in 


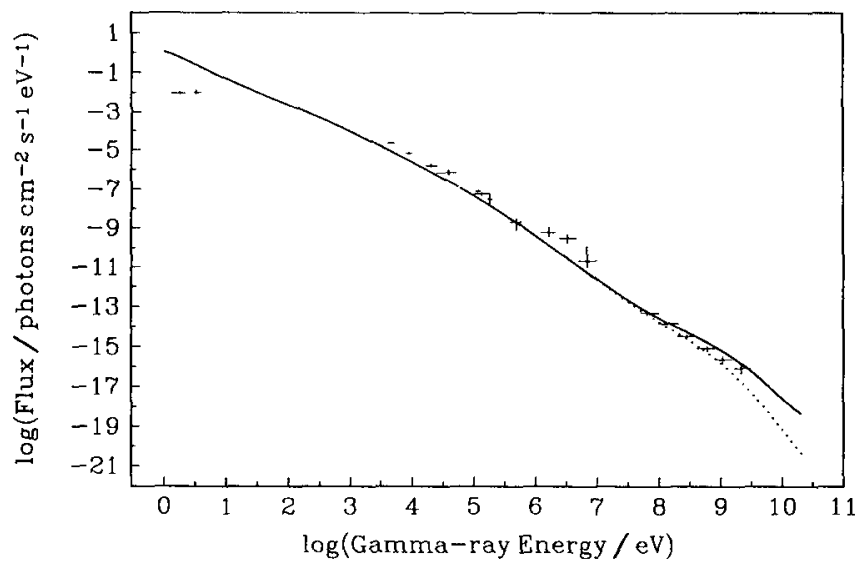

Fig. 2.- The calculated spectrum of the pulsed electromagnetic emission from the Crab pulsar (solid line) (Cheng, Ho, \& Ruderman 1986b). The dotted line is the modified spectrum of the pulsed electromagnetic emission from the Crab pulsar. For energy less than $3 \mathrm{MeV}$, the two spectra overlap. The observed data include those of Clear et al. (1987); Knight (1982); and Mahoney, Ling, \& Jacobson (1983).

different energy ranges. Synchrotron radiation dominates at lower energy than inverse-Compton scattering, with a cutoff at several MeV. Therefore the major contribution of the unpulsed gamma-ray spectrum comes from inverse-Compton scattering. Theoretically, the higher order generation of the unpulsed low-energy gamma rays will result in a spectrum of steeper slope than that of the lower order generations. Our calculation shows that the number of higher generation pairs is very small so the total unpulsed low-energy gamma-ray spectrum basically follows that of the first generation spectrum. We want to point out that our model unpulsed low-energy gamma-ray, keV-50 MeV, is much weaker than the corresponding observed unpulsed radiation. Therefore, the unpulsed radiation in this energy range must come from other mechanisms, e.g., synchrotron self-Compton mechanism.

The recent work of De Jager \& Harding (1992) on the highenergy gamma-ray spectrum of the Crab Nebula has marked

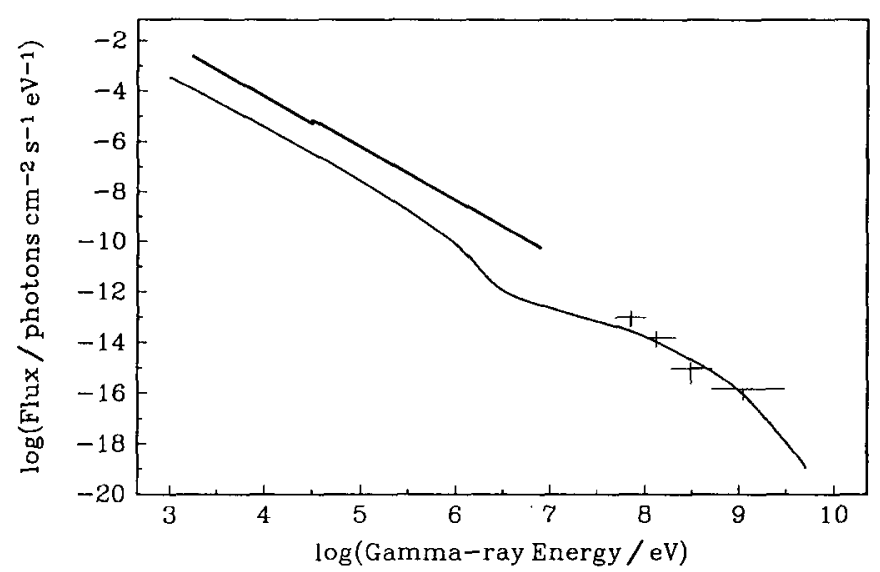

Fig. 3.-The calculated unpulsed gamma-ray spectrum from the Crab pulsar ( solid line). The observed data include those of Pravdo \& Serlemitsos (1981) and Walraven et al. (1975) (heavy solid line) and Clear et al. (1987) with their error estimates.

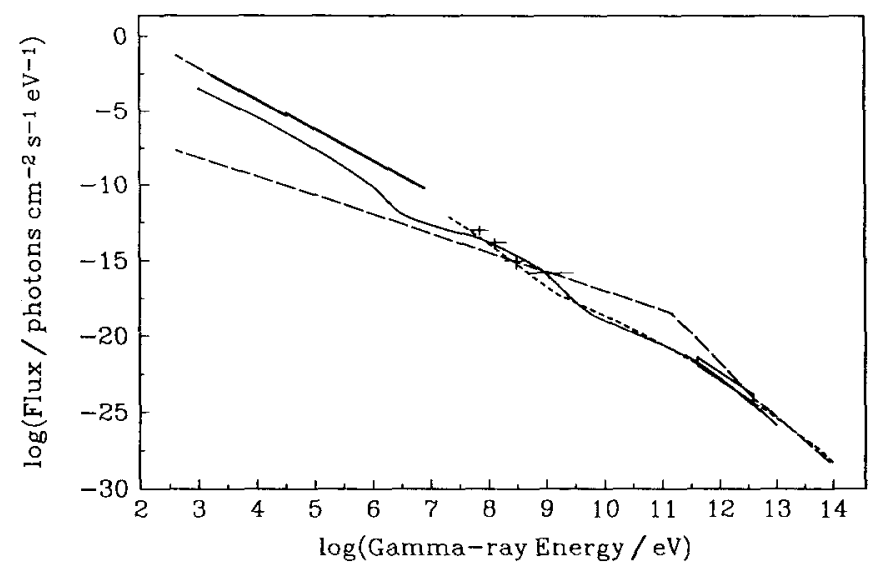

Fig. 4.-The results of different models for the unpulsed gamma rays from the Crab Nebula. The solid line is the result of our model. The dashed line is the result of the model by De Jager \& Harding (1992). The long dashed line is the Compton synchrotron model (Gould 1965). The spectra measured by $\operatorname{COS} B$ (Clear et al. 1987) (between $10 \mathrm{MeV}$ and $\mathrm{GeV}$ ), the Whipple group by Vacanti et al. (1991) (around a TeV), Walraven et al. (1975) (between $\mathrm{MeV}$ and $30 \mathrm{keV}$ ), and Pravdo \& Serlemitsos (1981) (below $30 \mathrm{keV}$ ) are also shown with their error estimates.

TABLE 1

COMParison of THE MOdel USEd IN THIS PAPER WITH THE MOdel BY DE JAGER AND HARDING

\begin{tabular}{|c|c|}
\hline Model Used in the Present Paper & $\begin{array}{l}\text { Model Used by De Jager } \\
\text { and Harding }\end{array}$ \\
\hline \multicolumn{2}{|c|}{ Input Physics } \\
\hline $\begin{array}{l}\text { Two different groups of } e^{ \pm} \text {pairs, } \\
\text { namely, } \\
\text { 1. X-rays }(\mathrm{MeV})-\gamma \text {-rays }(50 \\
\text { MeV-10 GeV) } \\
\text { 2. } \gamma \text {-rays }(0.1 \mathrm{TeV}-1 \mathrm{TeV})\end{array}$ & $\begin{array}{l}\text { Same group of } e^{ \pm} \text {pairs for } \\
\text { any range of radiation. }\end{array}$ \\
\hline $\begin{array}{l}\text { No further acceleration of the } e^{ \pm} \\
\text {pairs. They have already been } \\
\text { accelerated in the outer gap. }\end{array}$ & $\begin{array}{l}e^{ \pm} \text {pairs are accelerated by } \\
\text { shock acceleration } \\
\text { mechanism. }\end{array}$ \\
\hline \multicolumn{2}{|c|}{ Observational Predictions } \\
\hline $\begin{array}{l}\text { There are spectra cutoffs at different } \\
\text { energies. } \\
\text { 1. Cutoff of synchrotron radiation } \\
\text { spectrum at several } \mathrm{MeV} \text {. } \\
\text { 2. Cutoff of ICS spectrum at } \mathrm{TeV} \text {. }\end{array}$ & $\begin{array}{l}\text { The spectrum is } \\
\text { continuous in the } \\
\text { energy range of IR to } \\
10^{15} \mathrm{eV} \text {. }\end{array}$ \\
\hline $\begin{array}{l}\text { Angular size of the emission region } \\
\quad \approx 3 r_{L} / D \\
\quad \approx 4.5 \times 10^{8} / 6 \times 10^{21} \\
\quad \ll 1^{\prime \prime}\end{array}$ & $\begin{array}{l}\text { Angular size of the } \\
\text { emission region } \\
\approx 20^{\prime \prime}\end{array}$ \\
\hline $\begin{array}{l}\text { No more steady production of } \\
\text { photons at } 10 \mathrm{TeV} \text { or above } \\
\text { (because of magnetic pair } \\
\text { production). }\end{array}$ & $\begin{array}{l}\text { Production of photons is } \\
\text { predicted at } 10 \mathrm{TeV} \\
\text { and above. }\end{array}$ \\
\hline $\begin{array}{l}\text { More GeV photons are predicted (by } \\
\text { a factor of } 3 \text { ). }\end{array}$ & $\begin{array}{l}\text { Less GeV photons are } \\
\text { predicted. }\end{array}$ \\
\hline
\end{tabular}


differences with the model we propose here (cf. Fig. 4). As it is the other model currently used to calculate the high-energy gamma-ray spectrum of the Crab Nebula, it is worth comparing it with our model so that a clearer picture of the high-energy gamma-ray spectrum of the Crab Nebula emerges. The major differences are shown in Table 1.

As seen in the table, we can distinguish the two models from two different points of view, namely, the input physics and the observational predictions. In the input physics, the main differences rest on the electron/positron pairs. In our model, for different ranges of radiation, there are different groups of $e^{ \pm}$ pairs. There are two groups of $e^{ \pm}$pairs. The first group results from the collision between two pulsed photon beams beyond the light cylinder (this paper) and the second group results from the interactions between the pulsed photon beam and the gap primary electrons/positrons (cf. Paper I). The two groups radiate photons in the energy ranges (1) X-rays $(\mathrm{MeV})$ to $\gamma$ rays ( $50 \mathrm{MeV}$ to $10 \mathrm{GeV}$ ) and (2) $\gamma$-rays $(0.1 \mathrm{TeV}$ to $1 \mathrm{TeV})$. Moreover, these $e^{\ddagger}$ pairs undergo no further acceleration outside the outer gap. On the other hand, there is only one group of $e^{ \pm}$pairs for the whole range of radiation in the model of De Jager \& Harding. The $e^{ \pm}$pairs are also accelerated by the shock acceleration mechanism beyond the light cylinder.

Although the input physics of the two models differ markedly, we can hardly probe the true mechanism by direct evidence. Therefore, the observational prediction turns out to be the decisive element for the models. Let us compare the two models in this point of view. In our model, the spectrum has two different sharp cutoffs, at several $\mathrm{MeV}$ and at the $\mathrm{TeV}$ energy range respectively ( $\mathrm{cf}$. Fig. 4). The MeV cutoff is due to the synchrotron radiation spectrum, while the $\mathrm{TeV}$ cutoff is due to the inverse-Compton scattering. However, we remark that the sharp spectral break in $\mathrm{MeV}$ actually results from the fact that we have approximated the crossover regions by a single distance, but the more realistic model, in which the crossover region is extensive, should show a smoother spectrum around $\mathrm{MeV}$. Above $10 \mathrm{TeV}$, there is no steady production of photons because of the magnetic pair production. At $\mathrm{GeV}$ energies, our model predicts a higher flux (by a factor of about 3 ) than that of De Jager \& Harding's model. Their model also predicts a continuous spectrum in the energy range of IR to $10^{15} \mathrm{eV}$. The detailed differences are shown in Figure 4. In addition, the angular resolution of the emission source is much less than $1^{\prime \prime}$ in our model, while it is about $20^{\prime \prime}$ in the model of De Jager \& Harding (cf. Fig. 9 of De Jager \& Harding 1992).

Finally, we want to remark that Clear et al. (1987) report that there is some variability of the pulsed emission. According to our model, the unpulsed emission is directly related to the pulsed emission, so the intensity of the unpulsed emission should be correlated to that of the pulsed emission. However, there is one major unknown factor, namely, whether the variability of the pulsed emission results from the intrinsic intensity variation or the fluctuation of the beaming angle. If the variation is caused by the latter, then the intensity of the unpulsed emission may not be changed.

\section{REFERENCES}

Arons, J., Gallant, Y. A., Hoshino, M., Langdon, B., \& Max, C. E. 1991, in IAU Colloq. 128, Magnetospheric Structure and Emission Mechanisms of Radio Pulsars, ed. J. A. Gil, T. H. Hankins, \& J. M. Rankin, in press Blumenthal, G. R., \& Gould, R. J. 1970, Rev. Mod. Phys., 42, 237

Cheng, K. S., Ho, C., \& Ruderman, M. 1986a, ApJ, 300, 500(CHR I) 1986b, ApJ, 300, 522(CHR II)

Cheung, W. M., \& Cheng, K. S. 1993, ApJ, 413, 694 (Paper II)

Clear, J., et al. 1987, A\&A, 174, 85

Coroniti, F. V. 1990, ApJ, 349, 538

De Jager, O. C., \& Harding, A. K. 1992, ApJ, 396, 161

Gould, R. J. 1965, Phys. Rev. Lett., 15, 577

Grindlay, J. E., \& Hoffiman, J. A. 1971, Ap. Lett., 8, 209

Ho, C. 1989 , ApJ, 342, 396

Kennel, C. F., \& Coroniti, F. V. 1984, ApJ, 283, 694

Knight, F. K. 1982, ApJ, 260, 538

Kwok, P. W., Cheng, K. S., \& Lau, M. M. 1991, ApJ, 379, 653 (Paper I)

Mahoney, W. A., Ling, J. C., \& Jacobson, A. S. 1983, ApJ, 278, 784

Pravdo, S. H., \& Serlemitsos, P. 1981, ApJ, 246, 484

Rieke, G. H., \& Weekes, T. C. 1969, ApJ, 15, 577

Vacanti, G., et al. 1991, ApJ, 377, 467

Walraven, G. D., Hall, R. D., Meegan, C. A., Coleman, P. L., Shelton, D. H., \& Haymes, R. C. 1975, ApJ, 202, 502 